\title{
An Investigation of Bounds for the Regulator of Quadratic Fields
}

\author{
Michael J. Jacobson, Jr., Richard F. Lukes and Hugh C. Williams
}

\section{CONTENTS}

1. Introduction

2. Computation of $R$

3. Evaluation of $h$

4. The Cohen-Lenstra Heuristics

5. The size of $L\left(1, \chi_{\Delta}\right)$

6. Conclusion
It is well known that the nontorsion part of the unit group of a real quadratic field $\mathcal{K}$ is cyclic. With no loss of generality we may assume that it has a generator $\varepsilon_{0}>1$, called the fundamental unit of $\mathcal{K}$. The natural logarithm of $\varepsilon_{0}$ is called the regulator $R$ of $\mathcal{K}$. This paper considers the following problems: How large, and how small, can $R$ get? And how often?

The answer is simple for the problem of how small $R$ can be, but seems to be extremely difficult for the question of how large $R$ can get. In order to investigate this, we conducted several large-scale numerical experiments, involving the Extended Riemann Hypothesis and the Cohen-Lenstra class number heuristics. These experiments provide numerical confirmation for what is currently believed about the magnitude of $R$.

\section{INTRODUCTION}

Let $D$ denote a square-free integer and let $\mathcal{K}=$ $\mathbb{Q}(\sqrt{D})$ be the quadratic field formed by adjoining $\sqrt{D}$ to the rationals $\mathbb{Q}$. Set

$$
r= \begin{cases}2 & \text { if } D \equiv 1 \bmod 4 \\ 1 & \text { otherwise }\end{cases}
$$

Then $\Delta=(2 / r)^{2} D$ is the discriminant of $\mathcal{K}$. If

$$
\omega=\frac{1}{2}(\Delta+\sqrt{\Delta}),
$$

then

$$
\mathcal{O}=\mathbb{Z}+\omega \mathbb{Z}
$$

is the maximal order (the ring of algebraic integers) of $\mathcal{K}$. If $\alpha \in \mathcal{K}$ we denote, as usual, the norm of $\alpha$ by $N(\alpha)=\alpha \bar{\alpha}$, where $\bar{\alpha}$ is the conjugate of $\alpha$.

If $\mathcal{O}^{*}$ is the group of units in $\mathcal{O}$ and $\Delta>0$, we have $\mathcal{O}^{*}=\left\langle-1, \varepsilon_{0}\right\rangle$, for a uniquely determined $\varepsilon_{0}>1$, called the fundamental unit of $\mathcal{K}$. Let $R=\log \varepsilon_{0}$ denote the regulator of $\mathcal{K}$. Since $\varepsilon_{0} \in \mathcal{O}$, 
we have $\varepsilon_{0}=\frac{1}{2}(x+y \sqrt{\Delta})$, where $x, y \in \mathbb{Z}$. Also, since $\left|N\left(\varepsilon_{0}\right)\right|=\varepsilon_{0}\left|\bar{\varepsilon}_{0}\right|=1$, it is easy to see that

$$
\begin{aligned}
& \varepsilon_{0}-1<x<\varepsilon_{0}+1 \\
& \frac{\varepsilon_{0}-1}{\sqrt{\Delta}}<y<\frac{\varepsilon_{0}+1}{\sqrt{\Delta}} .
\end{aligned}
$$

Thus $x, y>0$, and the regulator provides us with a good estimate for the value of $\log x$ and $\log (\sqrt{\Delta} y)$. Because of the importance of the fundamental unit, particularly in characterizing all solutions of diophantine equations of the form $N(\alpha)=k$, where $\alpha \in \mathcal{O}$ and $k \in \mathbb{Z}$, it is of considerable interest to study the size of $R$.

When $\Delta=x^{2}+4$, where $2 \nmid x$, it is not difficult to show that $\varepsilon_{0}=\frac{1}{2}(x+\sqrt{\Delta})$. Thus, in this case, we have

$$
\varepsilon_{0}=\frac{1}{2}(\sqrt{\Delta-4}+\sqrt{\Delta})
$$

and

$$
R=\log \left(\frac{1}{2}(\sqrt{\Delta-4}+\sqrt{\Delta})\right) .
$$

In general, since $\varepsilon_{0}=\frac{1}{2}(x+y \sqrt{\Delta})$ with $x, y>0$ and $\left|\varepsilon_{0} \bar{\varepsilon}_{0}\right|=1$, we have $x=\sqrt{y^{2} \Delta \pm 4}$ and

$$
\varepsilon_{o}=\frac{\sqrt{y^{2} \Delta \pm 4}+y \sqrt{\Delta}}{2} \geq \frac{\sqrt{\Delta-4}+\sqrt{\Delta}}{2} .
$$

Hence

$$
R \geq \log \left(\frac{1}{2}(\sqrt{\Delta-4}+\sqrt{\Delta})\right) .
$$

Since $x^{2}+4$ is square-free infinitely often for odd $x$ (see [Nagell 1922], for example), we see that equality in (1.1) is achieved infinitely often. Consequently, we know just how small $R$ can be as a function of $\Delta$.

The question of how large $R$ can be is much more difficult. By a result of Hua [1982, p. 329], we can certainly say that

$$
R<\sqrt{\frac{1}{2} \Delta}\left(\frac{1}{2} \log \Delta+1\right),
$$

but this is not very near to a sharp bound like (1.1). Thus, we are left with two questions:

(1) What is the largest value that $R$ can attain as a function of $\Delta$ ?

(2) How often does $R$ become that large?
Both questions turn out to be extremely difficult, as we can see by examining the analytic class number formula

$$
2 R h=\sqrt{\Delta} L\left(1, \chi_{\Delta}\right) .
$$

Here $h$ is the class number of $\mathcal{K}$ and

$$
L\left(1, \chi_{\Delta}\right)=\lim _{s \rightarrow 1} L\left(s, \chi_{\Delta}\right),
$$

where the Dirichlet L-function is defined by

$$
L\left(s, \chi_{\Delta}\right)=\sum_{n=1}^{\infty} \frac{\chi_{\Delta}(n)}{n^{s}}=\prod_{p}\left(1-\frac{\chi_{\Delta}(p)}{p^{s}}\right)^{-1} .
$$

The character $\chi_{\Delta}$ here is the Kronecker symbol $(\Delta / n)$; the Euler product on the right of (1.3) is taken over all the primes $p$. Thus, in order for $R$ to be large it is necessary for $h$ to be small and $L\left(1, \chi_{\Delta}\right)$ to be large. How often $h$ can be small and how large (and how often) $L\left(1, \chi_{\Delta}\right)$ can be are very deep and difficult questions in number theory. For example, the famous Gauss Conjecture asserts that $h=1$ infinitely often, and the Extended Riemann Hypothesis (ERH) provides us with quite close bounds on $L\left(1, \chi_{\Delta}\right)$.

This article contains the results of some numerical experiments that we conducted in order to investigate problems (1) and (2). We first describe a large-scale computational trial that we implemented to verify the Cohen-Lenstra heuristics on the distribution of the odd part of the class number. We will next discuss further numerical experiments in which we attempted to see how closely the bounds of [Littlewood 1928] and [Shanks 1973] come to bracketing the value of $L\left(1, \chi_{\Delta}\right)$.

\section{COMPUTATION OF R}

The basic idea we used in our computation of $h$ was to first compute $R$ and then $L\left(1, \chi_{\Delta}\right)$ to sufficient accuracy that it is possible to use (1.2) to determine the integer $h$. In this section we discuss how we compute $R$ using a version of Lenstra's idea [1982], as described in [Mollin and Williams, p. 290]. 
The first step of this process is to estimate the value of $L\left(1, \chi_{\Delta}\right)$. Here, instead of using a truncated Euler product and Oesterlé's results [1979] to estimate the error as in [Mollin and Williams], we use an idea due to Bach [Bach 1994]. This is based on using a weighted average of truncated $\mathrm{Eu}-$ ler products to compute an approximation $S(Q, \Delta)$ of $\log L\left(1, \chi_{\Delta}\right)$ which, under the $\mathrm{ERH}$, has relative error $O(\log \Delta /(\sqrt{Q} \log Q))$. For some preselected value of $Q$ we compute

$$
C(Q)=\sum_{i=0}^{Q-1}(i+Q) \log (i+Q)=\sum_{i=Q}^{2 Q-1} i \log i
$$

and weights

$$
a_{j}=\frac{(Q+j) \log (Q+j)}{C(Q)}
$$

According to the explicit version of [Bach 1994, Theorem 9.2], under the ERH we have

$$
\left|\log L\left(1, \chi_{\Delta}\right)-\sum_{i=0}^{Q-1} a_{i} \log B(Q+i)\right| \leq A(Q, \Delta)
$$

where

$$
A(Q, \Delta)=\frac{A \log \Delta+B}{\log Q \sqrt{Q}}
$$

$A$ and $B$ can be determined, depending on the value of $Q$, by using Table 3 in [Bach 1994]. Also, $B(x)$ is defined by the truncated Euler product

$$
B(x)=\prod_{p<x}\left(1-\frac{(\Delta / p)}{p}\right)^{-1}
$$

where the product is taken over all primes $p<x$.

One of the real bottlenecks in computing estimates like

$$
S(Q, \Delta)=\sum_{i=0}^{Q-1} a_{i} \log B(Q+i)
$$

is the evaluation of the many Kronecker (Legendre) symbols $(\Delta / q)$. In order to accelerate this process, we first note the easily shown identity

$$
S(Q, \Delta)=\sum_{p \leq 2 Q-1} w(p) \log \left(1-\frac{(\Delta / p)}{p}\right)^{-1},
$$

where

$$
w(p)= \begin{cases}1 & \text { for } p<Q, \\ \sum_{p-Q+1}^{Q-1} a_{j} & \text { for } Q \leq p<2 Q-1 .\end{cases}
$$

Our technique of determining $S(Q, \Delta)$ consisted of computing and storing in a large table the quadratic residues and nonresidues and the values of $w(p) \log (p /(p-1))$ and $w(p) \log (p /(p+1))$ for all the primes $p \leq 10000$. We could then find the value of $w(p) \log (p /(p-(\Delta / p)))$ by little more than a single table look-up for each prime $p \leq 10000$; thus, we could easily evaluate

$$
S(Q, \Delta)=\sum_{p \leq 2 Q-1} w(p) \log \left(\frac{p}{p-(\Delta / p)}\right)
$$

and then compute an estimate of $L\left(1, \chi_{\Delta}\right)$ by a single exponentiation.

After conducting some preliminary experiments we found that a value of $Q=2000$ was very often sufficient (for $\Delta<10^{9}$ ) to estimate $L\left(1, \chi_{\Delta}\right.$ ) in order to establish $h=1$. This is a huge improvement over the truncated product method used in [Stephens and Williams 1988], where all primes less than 18000 had to be used in the estimate (compared with only 4000 using Bach's method). In fact, we found that using $Q=5000$ (i.e., primes less than 10000) was often sufficient to establish $h \leq 3$, and that this resulted in the best performance of our algorithm.

For fixed $Q$ and $\Delta$, put $E=\frac{1}{2} \sqrt{\Delta} \exp (S(Q, \Delta))$. Then $h R \approx E$. By using (1.2) and (2.1) we know (under the ERH) that

$$
|E-h R|<L^{2},
$$

where

$$
L^{2}=E \max \left\{e^{A(Q, \Delta)}-1,1-e^{-A(Q, \Delta)}\right\} .
$$


In order to get some indication of the growth rate of $L$ (for $Q=5000$ ), we evaluated it for prime radicands $D$ only, in various intervals: see Table 1 .

\begin{tabular}{|rrc|}
\hline interval & $\max (L)$ & $\operatorname{avg}(L)$ \\
\hline 1 & 26.01440 & 10.73694 \\
101 & 99.76966 & 50.64988 \\
201 & 120.47460 & 61.27755 \\
301 & 135.44843 & 68.64010 \\
401 & 146.94061 & 74.26657 \\
501 & 157.06318 & 78.86076 \\
601 & 166.13391 & 82.86471 \\
701 & 172.31836 & 86.53736 \\
801 & 176.91473 & 89.52843 \\
901 & 183.47702 & 92.59853 \\
1000 & 191.06620 & 95.27484 \\
\hline
\end{tabular}

TABLE 1. Growth of $L$. Here and throughout the article, "interval $i$ " is the set of all prime values of $D$ such that $(i-1) \times 10^{6}<D<i \times 10^{6}$. The second and third columns give the maximum and average values of $L$ found in each interval.

With the value of $L$ computed above we calculated the regulator by using the modified version of the second algorithm in [Mollin and Williams, $\S 7$ ]. This algorithm determines a value for $h^{*} R<E+$ $L^{2}$, where $h^{*}$ is some integer. It then finds the value of $h^{*}$ and thus $R$. In particular, if $R<E / \sqrt{L}$, this algorithm will determine $R$ quickly. However, usually we have $R \geq E / \sqrt{L}$. In this case the set of all primes $q_{1}=2, q_{2}=3, \ldots, q_{n}<B=\sqrt{L}+L^{2} \sqrt{L} / E$ must be computed. It is then necessary to check for each of these primes $q<B$ whether any reduced principal ideal $\mathfrak{a}$ at a distance from $\mathfrak{a}_{1}=(1)$ very close to $h^{*} R / q$ is such that $\mathfrak{a}=\mathfrak{a}_{1}$. If so, $q$ divides $h^{*}$; otherwise it doesn't. If $q \mid h^{*}$ we must also check the reduced principal ideals at distance $h^{*} R / q^{2}, h^{*} R / q^{3}$, etc., until we find one equal to $\mathfrak{a}_{1}$ at distance close to $h^{*} R / q^{\alpha}$, but we do not find any at distance close to $h^{*} R / q^{\alpha+1}$. Then $q^{\alpha}$ exactly divides $h^{*}$ : in symbols, $q^{\alpha} \| h^{*}$. Since $h^{*}<B$, we must ultimately find

$$
h^{*}=\prod_{i=1}^{n} q_{i}^{\alpha_{i}} .
$$

Of course, if we find that $q^{\alpha} \| h^{*}$, then $h^{*} / q^{\alpha}<$ $B / q^{\alpha}$, allowing us to replace $B$ by $B / q^{\alpha}$.

It was this latter process that we modified. For each prime $q_{u}<B$, instead of finding a reduced principal ideal $\mathfrak{a}_{m}$ such that $\delta_{m}$, the distance of $\mathfrak{a}_{m}$ from $\mathfrak{a}_{1}$ [Mollin and Williams, p. 285], is such that $\delta_{m} \approx h^{*} R / q_{u}$, we determine a reduced principal ideal $\mathfrak{a}_{j_{u}}$ such that

$$
\frac{h^{*} R}{q_{u}}<\delta_{j_{u}}<\frac{h^{*} R}{q_{u}}+\delta_{t} .
$$

Here $\delta_{t}$ is that distance such that $\delta_{t}<L<\delta_{t+1}$. We next produce a list $\mathcal{J}$ of reduced principal ideals $\mathfrak{a}_{t_{0}}, \mathfrak{a}_{t_{1}}, \mathfrak{a}_{t_{2}}, \ldots, \mathfrak{a}_{t_{m}}$ such that $\mathfrak{a}_{t_{0}}=\mathfrak{a}_{t}, \delta_{t_{k}} \approx 2 \delta_{t_{k-1}}$ and

$$
\delta_{t_{m-1}}<\frac{1}{2} h^{*} R<\delta_{t_{m}} .
$$

In order to determine $h^{*} R$, the list $\mathcal{T}$ made up of each reduced principal ideal $\mathfrak{a}_{k}$ and its distance $\delta_{k}$ such that $\delta_{k}<L$ had to be computed and stored; hence, we may assume that this list is still in existence. If $q_{u}$ divides $h^{*}$, then $\mathfrak{a}_{j_{u}}$ must be in $\mathcal{T}$ and

$$
\delta_{j_{u}}=\frac{h^{*} R}{q_{u}}+\delta_{k}
$$

when $\mathfrak{a}_{j_{u}}=\mathfrak{a}_{k}$. If, from the next prime, we have an ideal $\mathfrak{a}_{j_{u+1}}$ such that

$$
\frac{h^{*} R}{q_{u+1}}<\delta_{j_{u+1}}<\frac{h^{*} R}{q_{u+1}}+\delta_{t},
$$

we notice that, if we have a reduced principal ideal $\mathfrak{a}_{i_{u}}$ with distance $\delta_{i_{u}}$ such that

$$
\begin{aligned}
& \delta_{i_{u}} \approx \frac{h^{*} R}{q_{u}}-\delta_{j_{u+1}}, \\
& \delta_{i_{u}}<\frac{h^{*} R}{q_{u}}-\delta_{j_{u+1}}
\end{aligned}
$$

and

$$
\frac{h^{*} R}{q_{u}}<\delta_{i_{u}}+\delta_{j_{u+1}}<\frac{h^{*} R}{q_{u}}+\delta_{t},
$$

we can then set $\mathfrak{a}_{j_{u}}$ to be a reduced ideal equivalent to $\mathfrak{a}_{i_{u}} \mathfrak{a}_{j_{u+1}}$ with $\delta_{j_{u}} \approx \delta_{i_{u}}+\delta_{j_{u+1}}$ and

$$
\frac{h^{*} R}{q_{u}}<\delta_{j_{u}}<\frac{h^{*} R}{q_{u}}+\delta_{t}
$$


Now suppose we let $h^{*} R / q_{u}-\delta_{j_{u+1}}=\rho \delta_{t}$, and put $s=\lfloor\rho\rfloor+1$. If we represent $s$ in binary as

$$
s=b_{r} 2^{r}+b_{r-1} 2^{r-1}+\ldots+b_{o},
$$

where $b_{r}=1$ and $b_{j}=0,1$ for $j=0,1,2, \ldots, r-1$, then

$$
s \delta_{t}=b_{r} 2^{r} \delta_{t}+b_{r-1} 2^{r-1} \delta_{t}+\cdots+b_{o} \delta_{t} .
$$

In our list $\mathcal{J}$ we have $\delta_{t_{k}} \approx 2 \delta_{t_{k-1}}$, so we can find $\mathfrak{a}_{i_{u}}$ with distance $\delta_{i_{u}} \approx \rho \delta_{t}$ by simply computing a reduced ideal equivalent to

$$
\prod_{\substack{j=0 \\ b_{j}=1}}^{r} \mathfrak{a}_{t_{j}}^{b_{j}} .
$$

Thus, starting with $u=n$, we first find a reduced principal ideal $\mathfrak{a}_{j_{u}}$ with distance $\delta_{j_{u}} \approx E / q_{n} ;$ we can then determine $\mathfrak{a}_{j_{u-1}}, \mathfrak{a}_{j_{u-2}}, \ldots$ by the method described above. Whenever we get $\mathfrak{a}_{j_{v}}=\mathfrak{a}_{k}$, where $\mathfrak{a}_{k} \in \mathcal{T}$, then $q_{v}$ divides $h^{*}$. We then replace $E$ by $E / q_{v}$ and $B$ by $B / q_{v}$ and repeat the process, starting at $q_{v}$, until we find $\alpha$ such that $q_{v}^{\alpha} \| h^{*}$. When this procedure has been done for all primes $q_{1}, q_{2}, \ldots, q_{n}<B$ or $B=1$, we will have $h^{*}$.

To ensure that this modified algorithm is in fact faster than the unmodified algorithm or even Algorithm 7.1 of [Mollin and Williams], we programmed all three in C and ran them on an IBM RS6000/590 workstation. Algorithm 7.1 computes $R$ with time complexity $O\left(D^{1 / 4+\varepsilon}\right)$; the unmodified algorithm mentioned above and the modified version both execute in time $O\left(D^{1 / 5+\varepsilon}\right)$ under the ERH. In both of these cases the computed value of $R$ is provably correct; the ERH is needed only for the complexity estimate. The modified version was always faster than the unmodified version, and except for the smallest values of $D$ was the fastest overall. Algorithm 7.1 was the best for small $D$.

\section{EVALUATION OF $h$}

For a given $D$ with $Q=5000$, put

$$
\tilde{h}=\operatorname{round}\left(\frac{\sqrt{\Delta} \exp (S(Q, \Delta))}{2 R}\right) \text {, }
$$

where by round $(x)$ we denote the nearest integer to $x$. When $\tilde{h}$ is large, say $\tilde{h}>D^{1 / 8}$, it is often very time-consuming to produce a new value for $S(Q, \Delta)$ (with a larger $Q$ value) such that

$$
h=\operatorname{round}\left(\frac{\sqrt{\Delta} \exp (S(Q, \Delta))}{2 R}\right) .
$$

This problem can, to a very large extent, be overcome by first finding a factor $h_{1}$ of $h$ such that $h / h_{1}$ is small.

Since, by the heuristics of Cohen and Lenstra $[1983 ; 1984]$, we expect that the class group of $\mathcal{K}$ is very frequently cyclic, finding such an $h_{1}$ is usually not very difficult. We simply select an ideal $\mathfrak{a}$ lying over a prime $q$ where $(D / q)=1$. We then compute a reduced ideal $\mathfrak{b} \sim \mathfrak{a}^{\tilde{h}}$. Often $\mathfrak{b} \sim(1)$, in which case we can put $m=\tilde{h}$. If $\mathfrak{b} \nsim(1)$, we compute $\mathfrak{b}_{i} \sim \mathfrak{b} \mathfrak{a}^{i}, \mathfrak{b}_{-i} \sim \mathfrak{b} \overline{\mathfrak{a}}^{i}$ until we find $\mathfrak{b}_{i} \sim(1)$ or $\mathfrak{b}_{-i} \sim$ (1). In the first case we put $m=\tilde{h}+i$ and in the second we put $m=\tilde{h}-i$.

Since we were confining our attention to fields with $D<10^{9}$, we were able to check for ideal principality by searching an ordered list of all the reduced principal ideals. This technique was feasible because fields with $\tilde{h}$ relatively large (say $\tilde{h}>3$ ) have relatively few principal ideals.

The value of $m$ here is very often the class number; however, we must search over all the divisors of $m$ to find the least $k$ such that $\mathfrak{a}^{k} \sim(1)$. We now know that $k$ divides $h$. If $k$ is too small, we repeat the above process for other prime ideals and take as our value of $h_{1}$ the least common multiple of all the $k$ values that we find. We did this until we found $h_{1}>\frac{1}{3} \tilde{h}$. This was possible in all but a few cases which were handled separately. We seldom had to use more than one trial ideal, but occasionally as many as 12 were needed.

We also experimented with using $h^{*}$ instead of $\tilde{h}$. For fields with large $h$, the value of $h^{*}$ is usually a better approximation to $h$ than $\tilde{h}$; thus, fewer ideal multiplications are needed to find $m$. However, when $h$ is large, often $R$ is determined immediately from the list $\mathcal{T}$ [Mollin and Williams] and 
$h^{*}$ is never evaluated. Hence no significant savings occurred on using $h^{*}$ instead of $\tilde{h}$.

Once the regulator $R$ and a value for $h_{1}>\tilde{h} / 3$ had been determined, we used Algorithm 3.1 to find $h$.

Algorithm 3.1 (Class Number of $\mathbb{Q}(\sqrt{D})$ ). Input: $\Delta$, the discriminant of a real quadratic field.

Output: $h$ and $R$.

- Set $Q=5000$. Compute $S(Q, \Delta), R$, and $h_{1}$ as described above.

- Repeat:

- Compute $F=\sqrt{\Delta} \exp (S(Q, \Delta)) /\left(2 R h_{1}\right)$.

- Set $\tilde{h}_{2}=\operatorname{round}(F)$ and $\kappa=F-\tilde{h}_{2}$.

- If $A(Q, \Delta)<\log \left(\left(\tilde{h}_{2}+1\right) /\left(\tilde{h}_{2}+|\kappa|\right)\right)$, output $h=\tilde{h}_{2} h_{1}$ and terminate; otherwise, set $Q=$ $Q+5000$, recompute $S(Q, \Delta)$, and return to beginning of loop.

Only very rarely did we have to go beyond the $Q=5000$ used in the initial approximation to $\log L\left(1, \chi_{\Delta}\right)$ : typically, for less than 10 out of approximately 50000 fields examined in each interval, as compared to less than 120 fields using truncated Euler products with $Q=18000$. A more significant improvement is the maximum $Q$ values required in an interval, which are much smaller than those required by the truncated product method. This is important because Bach's method requires the whole approximation to be recomputed in these cases, whereas a truncated product approximation can be improved simply by adding more terms. However, since we rarely require more accuracy and, if we do, the $Q$ value needed is usually fairly small, our algorithm still runs faster using Bach's method. In these cases we used the usual Jacobi algorithm to evaluate the Legendre symbols $(\Delta / q)$. We emphasize here that the values of these class numbers are dependent on the truth of the ERH; however, given the discussion in [Shanks 1971], it would be a most unusual event, should the ERH be false, for any of the class numbers computed by this technique to be incorrect, assuming that the calculations are carried out correctly.
The algorithms for determining $h_{1}$ and $h$ were also coded in C and run on an IBM RS6000/590 workstation. Using Bach's method, our algorithms executed about 1.5 times as quickly as they did using the truncated Euler product method.

\section{THE COHEN-LENSTRA HEURISTICS}

Let $\boldsymbol{G}$ be the class group of $\mathcal{K}$ and let $\boldsymbol{G}^{*}$ be the odd part of $\boldsymbol{G}$. Cohen and Lenstra $[1983 ; 1984]$ provide some heuristics on the distribution of various $G^{*}$. For example, if we define

$$
w(n)=\prod_{p^{\alpha} \| n} \frac{1}{p^{\alpha}(1-1 / p)\left(1-1 / p^{2}\right) \ldots\left(1-1 / p^{\alpha}\right)},
$$

the probability that $h^{*}=\left|\boldsymbol{G}^{*}\right|$ is equal to $k$ is

$$
\operatorname{Prob}\left(h^{*}=k\right)=\frac{C w(k)}{k},
$$

where $C=.754458173 \ldots$ Since $w(1)=1$, we see that this result would predict that $h^{*}=1$ about $75 \%$ of the time, a figure supported by the computations in [Stephens and Williams 1988]. In fact, under this heuristic we would expect that the probability that $h^{*}$ exceeds $x$ is

$$
\operatorname{Prob}\left(h^{*}>x\right)=C \sum_{\substack{j>x \\ j \text { odd }}}^{\infty} \frac{w(j)}{j}
$$

Now, if we put

$$
W(x)=\sum_{\substack{n>x \\ n \text { odd }}} w(n)
$$

we can use standard analytic methods such as those employed in [Landau 1936] to show that there exist constants $E_{1}$ and $E_{2}$ such that

$$
W(x)=E_{1} \log x+E_{2}+O\left(\frac{\log x}{x}\right)
$$


where

$$
\begin{aligned}
E_{1} & =(2 C)^{-1}=\eta_{\infty}(2) C_{\infty}, \\
C_{\infty} & =\prod_{j=1}^{\infty} \zeta(j+1)=2.294856589 \ldots, \\
\eta_{\infty}(2) & =\prod_{i=1}^{\infty}\left(1-2^{-i}\right)=.288788095 \ldots
\end{aligned}
$$

By using partial summation on (4.2) and the result of (4.3) we get

$$
\operatorname{Prob}\left(h^{*}>x\right)=\frac{1}{2 x}+O\left(\frac{\log x}{x^{2}}\right) .
$$

Thus, under the Cohen-Lenstra heuristics we'd expect that $h^{*}$ is most likely to be small. Since $\operatorname{Prob}\left(h^{*}=1\right) \approx \frac{3}{4}$, we will write this as

$$
1-\operatorname{Prob}\left(h^{*} \leq x\right)=\frac{1}{2 x+2}+O\left(\frac{\log x}{x^{2}}\right) .
$$

Thus we would expect that

$$
k+1=\frac{1}{2}\left(\frac{1}{1-\operatorname{Prob}\left(h^{*} \leq k\right)}\right)+O\left(\frac{\log k}{k^{2}}\right),
$$

a result that can be used to test the accuracy of (4.4).

Let $h(p)$ be the class number of the field $\mathbb{Q}(\sqrt{D})$, where $p$ is a prime. By using some further assumptions, Cohen was able to show that

$$
\sum_{\substack{p \leq x \\ p \equiv 1 \bmod 4}} h(p) \sim \frac{1}{8} x,
$$

a result conjectured by Hooley [1984] at about the same time.

In order to test the validity of (4.1), (4.5) and (4.6), we computed all the class numbers for all the fields $\mathbb{Q}(\sqrt{D})$ where $D<10^{8}$ and all the fields $\mathbb{Q}(\sqrt{p})$ where $p$ is a prime up to $10^{9}$. This computation of over $10^{8}$ class numbers required just under four weeks on the DECstation 5000/200. In order to describe its results, we introduce some notation.

For a finite group $\boldsymbol{G}$ we define

$$
f_{k}(\boldsymbol{G})=\left\{\begin{array}{ll}
1 & \text { when }|\boldsymbol{G}|=k, \\
0 & \text { otherwise }
\end{array} .\right.
$$

Let $D$ denote any square-free positive integer, and let $\boldsymbol{G}^{*}(D)$ represent the odd part of the class group of $\mathbb{Q}(\sqrt{D})$. Put

$$
\begin{aligned}
& \mathcal{D}_{1}(x)=\{D \leq x \mid D \equiv 1 \bmod 4\} \\
& \mathcal{D}_{2}(x)=\{D \leq x \mid D \not \equiv 1 \bmod 4\} \\
& \mathcal{P}_{1}(x)=\{p \leq x \mid p \equiv 1 \bmod 4, p \text { prime }\} \\
& \mathcal{P}_{2}(x)=\{p \leq x \mid p \equiv 3 \bmod 4, p \text { prime }\} .
\end{aligned}
$$

For each $\mathcal{D}(x) \in\left\{\mathcal{D}_{1}(x), \mathcal{D}_{2}(x), \mathcal{P}_{1}(x), \mathcal{P}_{2}(x)\right\}$, define

$$
\begin{aligned}
r_{i}(x) & =\frac{\sum_{D \in \mathcal{D}(x)} f_{i}\left(\boldsymbol{G}^{*}(D)\right)}{\sum_{D \in \mathcal{D}(x)} 1}, & & q_{i}(x)=\frac{r_{i}(x) i}{C w(i)}, \\
t_{i}(x) & =\frac{1}{2}\left(\frac{1}{1-s_{i}(x)}\right), & s_{i}(x) & =\sum_{j \leq i} r_{i}(x) .
\end{aligned}
$$

Also, put

$$
H^{*}(x)=\sum_{d \in \mathcal{D}(x)} h^{*}(D)
$$

Tables 2 and 3 provide values of $q_{i}(x)$ for various choices of $i$ and $x$ for $\Delta \equiv 1 \bmod 4, \Delta<10^{8}$ and for $\Delta=p \equiv 1 \bmod 4$ and $p<10^{9}$. The corresponding tables for $\mathcal{D}(x)=\mathcal{D}_{2}(x)$ and $\mathcal{P}_{2}(x)$ are so similar that in the interest of brevity we do not include them here. Tables 4 and 5 provide values of $t_{i}(x)$ for various choices of $i$ and $x$ and $\mathcal{D}(x)=\mathcal{D}_{1}(x)$ and $\mathcal{P}_{1}(x)$. Again, because of the similarity of the corresponding tables for $\mathcal{D}(x)=\mathcal{D}_{2}(x)$ and $\mathcal{P}_{2}(x)$, we do not include them here. Finally, Table 6 provides values for $H^{*}(x)$ and $8 H^{*}(x) / x$ for $\mathcal{D}(x)=\mathcal{P}_{1}(x)$. The table for $\mathcal{D}(x)=\mathcal{P}_{2}(x)$ is very similar.

Notice that all of these results provide numerical support for the Cohen-Lenstra heuristics, and in particular that small values of $h^{*}$ seem to occur infinitely often, even when we restrict the radicands of the fields to prime values. In these cases, of course, we have $h=h^{*}$. 


\begin{tabular}{|rccccccc|}
\hline$x$ & $q_{1}(x)$ & $q_{3}(x)$ & $q_{5}(x)$ & $q_{7}(x)$ & $q_{9}(x)$ & $q_{11}(x)$ & $q_{27}(x)$ \\
\hline 1000000 & 1.06119 & 0.85263 & 0.95644 & 0.94918 & 0.70424 & 0.90228 & 0.47347 \\
10000000 & 1.03676 & 0.89604 & 0.99125 & 0.99564 & 0.83023 & 0.97519 & 0.69086 \\
20000000 & 1.03178 & 0.90683 & 0.99465 & 1.00142 & 0.84625 & 0.98812 & 0.74718 \\
30000000 & 1.02923 & 0.91246 & 0.99592 & 1.00250 & 0.85705 & 0.99247 & 0.76587 \\
40000000 & 1.02752 & 0.91613 & 0.99663 & 1.00194 & 0.86264 & 0.99791 & 0.78753 \\
50000000 & 1.02634 & 0.91893 & 0.99664 & 1.00315 & 0.86638 & 0.99846 & 0.79660 \\
60000000 & 1.02541 & 0.92078 & 0.99588 & 1.00446 & 0.87092 & 0.99982 & 0.80705 \\
70000000 & 1.02461 & 0.92235 & 0.99632 & 1.00504 & 0.87567 & 1.00148 & 0.81494 \\
80000000 & 1.02389 & 0.92374 & 0.99637 & 1.00623 & 0.87874 & 1.00372 & 0.82014 \\
90000000 & 1.02333 & 0.92480 & 0.99702 & 1.00608 & 0.88182 & 1.00418 & 0.82863 \\
100000000 & 1.02284 & 0.92605 & 0.99695 & 1.00581 & 0.88409 & 1.00528 & 0.83205 \\
\hline
\end{tabular}

TABLE 2. Values of $q_{i}(x)$ for $\Delta \equiv 1 \bmod 4$.

\begin{tabular}{|rccccccc|}
\hline$x$ & $q_{1}(x)$ & $q_{3}(x)$ & $q_{5}(x)$ & $q_{7}(x)$ & $q_{9}(x)$ & $q_{11}(x)$ & $q_{27}(x)$ \\
\hline 1000000 & 1.03912 & 0.87049 & 0.98999 & 1.05015 & 0.74868 & 0.89694 & 0.80228 \\
10000000 & 1.02286 & 0.91026 & 1.00832 & 1.00988 & 0.89654 & 1.00820 & 0.83991 \\
20000000 & 1.01992 & 0.91885 & 1.01125 & 1.01036 & 0.89047 & 1.00770 & 0.87678 \\
30000000 & 1.01878 & 0.92317 & 1.00562 & 1.02080 & 0.89756 & 1.00138 & 0.88219 \\
40000000 & 1.01746 & 0.92762 & 1.00621 & 1.02143 & 0.89815 & 1.01307 & 0.89369 \\
50000000 & 1.01679 & 0.93026 & 1.00793 & 1.01899 & 0.90235 & 1.01437 & 0.89445 \\
60000000 & 1.01614 & 0.93257 & 1.00686 & 1.01727 & 0.90852 & 1.01408 & 0.90140 \\
70000000 & 1.01563 & 0.93519 & 1.00600 & 1.01803 & 0.91051 & 1.01274 & 0.90768 \\
80000000 & 1.01515 & 0.93662 & 1.00488 & 1.01891 & 0.91308 & 1.01263 & 0.90514 \\
90000000 & 1.01493 & 0.93712 & 1.00600 & 1.01489 & 0.91691 & 1.01078 & 0.89925 \\
100000000 & 1.01468 & 0.93864 & 1.00478 & 1.01335 & 0.91944 & 1.00665 & 0.90274 \\
200000000 & 1.01314 & 0.94558 & 1.00057 & 1.01216 & 0.92337 & 1.00713 & 0.90869 \\
300000000 & 1.01241 & 0.94866 & 1.00118 & 1.00676 & 0.92586 & 1.00590 & 0.91010 \\
400000000 & 1.01169 & 0.95144 & 1.00229 & 1.00406 & 0.92779 & 1.00362 & 0.91560 \\
500000000 & 1.01122 & 0.95334 & 1.00100 & 1.00519 & 0.93096 & 1.00409 & 0.91528 \\
600000000 & 1.01077 & 0.95493 & 1.00120 & 1.00534 & 0.93239 & 1.00461 & 0.92144 \\
700000000 & 1.01045 & 0.95583 & 1.00199 & 1.00608 & 0.93323 & 1.00523 & 0.92348 \\
800000000 & 1.01020 & 0.95683 & 1.00179 & 1.00619 & 0.93468 & 1.00506 & 0.92527 \\
900000000 & 1.00998 & 0.95777 & 1.00186 & 1.00629 & 0.93499 & 1.00509 & 0.92732 \\
1000000000 & 1.00976 & 0.95830 & 1.00239 & 1.00646 & 0.93604 & 1.00508 & 0.92706 \\
\hline
\end{tabular}

TABLE 3. Values of $q_{i}(x)$ for $p \equiv 1 \bmod 4$.

\section{THE SIZE OF $L(1, \chi)$}

Littlewood [1928] and Shanks [1973] have shown that, under the ERH, we have

$$
\begin{aligned}
(1+o(1))\left(c_{1} \log \log \Delta\right)^{-1} & <L\left(1, \chi_{\Delta}\right) \\
& <(1+o(1)) c_{2} \log \log \Delta,
\end{aligned}
$$

where $c_{1}$ and $c_{2}$ depend upon the parity of $\Delta$ :

$$
\begin{aligned}
& c_{1}=12 e^{\gamma} / \pi^{2} \quad \text { and } \quad c_{2}=2 e^{\gamma} \quad \text { when } 2 \nmid \Delta \text {, } \\
& c_{1}=8 e^{\gamma} / \pi^{2} \text { and } \quad c_{2}=e^{\gamma} \quad \text { when } 2 \mid \Delta \text {. }
\end{aligned}
$$

In his numerical examination of (5.1), Shanks [1973] defined for a fixed $\Delta$ the upper and lower Littlewood indices as

$$
\begin{aligned}
& \mathrm{ULI}=L\left(1, \chi_{\Delta}\right) /\left(c_{2} \log \log \Delta\right), \\
& \mathrm{LLI}=L\left(1, \chi_{\Delta}\right) c_{1} \log \log \Delta .
\end{aligned}
$$




\begin{tabular}{|rcccrccc|}
\hline \multicolumn{1}{|c}{$x$} & $t_{1}(x)$ & $t_{3}(x)$ & \multicolumn{1}{c}{$t_{5}(x)$} & \multicolumn{1}{c|}{$t_{7}(x)$} & $t_{9}(x)$ & $t_{11}(x)$ & \multicolumn{1}{c|}{$t_{27}(x)$} \\
\hline 1000000 & 2.50786 & 5.42530 & 8.91565 & 12.81041 & 17.88166 & 22.96408 & 109.6509 \\
10000000 & 2.29561 & 4.75574 & 7.38079 & 10.02841 & 13.58368 & 16.60010 & 55.01249 \\
20000000 & 2.25667 & 4.64952 & 7.14116 & 9.61024 & 12.91103 & 15.64977 & 48.43620 \\
30000000 & 2.23723 & 4.59746 & 7.02378 & 9.40226 & 12.59204 & 15.19731 & 45.43814 \\
40000000 & 2.22443 & 4.56286 & 6.94593 & 9.26159 & 12.36781 & 14.88841 & 43.53718 \\
50000000 & 2.21560 & 4.54032 & 6.89384 & 9.17287 & 12.22767 & 14.68742 & 42.23651 \\
60000000 & 2.20874 & 4.52115 & 6.84708 & 9.09414 & 12.10904 & 14.52054 & 41.36938 \\
70000000 & 2.20287 & 4.50462 & 6.81076 & 9.03188 & 12.02043 & 14.39801 & 40.61104 \\
80000000 & 2.19765 & 4.48987 & 6.77728 & 8.97656 & 11.93639 & 14.28389 & 39.94645 \\
90000000 & 2.19354 & 4.47810 & 6.75275 & 8.93314 & 11.87337 & 14.19501 & 39.48465 \\
100000000 & 2.18998 & 4.46955 & 6.73308 & 8.89798 & 11.82131 & 14.12367 & 39.02412 \\
\hline
\end{tabular}

TABLE 4. Values of $t_{i}(x)$ for $\Delta \equiv 1 \bmod 4$.

\begin{tabular}{|rccccccc|}
\hline$x$ & $t_{1}(x)$ & $t_{3}(x)$ & $t_{5}(x)$ & $t_{7}(x)$ & $t_{9}(x)$ & $t_{11}(x)$ & $t_{27}(x)$ \\
\hline 1000000 & 2.31449 & 4.69162 & 7.22253 & 9.92777 & 12.95470 & 15.41109 & 55.48867 \\
10000000 & 2.19018 & 4.39240 & 6.59663 & 8.67220 & 11.47744 & 13.64301 & 36.38335 \\
20000000 & 2.16904 & 4.34869 & 6.50789 & 8.52074 & 11.18966 & 13.23712 & 34.28533 \\
30000000 & 2.16105 & 4.33701 & 6.46395 & 8.47241 & 11.13404 & 13.14434 & 33.69292 \\
40000000 & 2.15178 & 4.32065 & 6.42954 & 8.41497 & 11.03731 & 13.03696 & 32.98149 \\
50000000 & 2.14711 & 4.31417 & 6.42053 & 8.39339 & 11.01621 & 13.01053 & 32.63839 \\
60000000 & 2.14260 & 4.30673 & 6.40077 & 8.35532 & 10.97404 & 12.95108 & 32.35346 \\
70000000 & 2.13905 & 4.30460 & 6.39342 & 8.34469 & 10.96322 & 12.93295 & 32.21378 \\
80000000 & 2.13576 & 4.29791 & 6.37521 & 8.31590 & 10.92319 & 12.87703 & 32.04356 \\
90000000 & 2.13419 & 4.29391 & 6.36986 & 8.29684 & 10.90465 & 12.84707 & 31.84695 \\
100000000 & 2.13247 & 4.29399 & 6.36629 & 8.28698 & 10.89705 & 12.82721 & 31.69778 \\
200000000 & 2.12197 & 4.28339 & 6.33025 & 8.22313 & 10.80130 & 12.69580 & 30.99612 \\
300000000 & 2.11706 & 4.27754 & 6.31934 & 8.19169 & 10.75619 & 12.63083 & 30.63904 \\
400000000 & 2.11217 & 4.27035 & 6.30698 & 8.16446 & 10.71624 & 12.57083 & 30.40868 \\
500000000 & 2.10902 & 4.26615 & 6.29396 & 8.14535 & 10.69473 & 12.54227 & 30.24493 \\
600000000 & 2.10600 & 4.26107 & 6.28353 & 8.12824 & 10.67037 & 12.50989 & 30.15319 \\
700000000 & 2.10388 & 4.25650 & 6.27594 & 8.11728 & 10.65447 & 12.48937 & 30.07765 \\
800000000 & 2.10222 & 4.25425 & 6.27045 & 8.10837 & 10.64429 & 12.47502 & 30.02542 \\
900000000 & 2.10075 & 4.25251 & 6.26689 & 8.10267 & 10.63557 & 12.46310 & 29.98853 \\
1000000000 & 2.09927 & 4.24886 & 6.26053 & 8.09245 & 10.62169 & 12.44402 & 29.92182 \\
\hline
\end{tabular}

TABLE 5. Values of $t_{i}(x)$ for $p \equiv 1 \bmod 4$.

If (5.1) is true, then as $\Delta$ increases, we would expect that extreme values of the ULI and LLI would tend to approach 1.

In fact, Chowla [1949] has shown that, for any positive $\varepsilon<1$, the inequalities ULI $>\frac{1}{2}(1-\varepsilon)$ and LLI $<2(1-\varepsilon)$ hold, each for an infinite sequence of values of $\Delta$. Furthermore, Joshi [1970] showed that, if $c$ and $d$ are relatively prime positive integers and $8 \mid d$, then as $\Delta$ runs through prime values congruent to $c \bmod d$, we have

$$
\mathrm{ULI}>\frac{1-\varepsilon}{2} \prod_{p \mid d} \frac{1-1 / p}{1-(p / c) / p}
$$

and

$$
\mathrm{LLI}<2(1-\varepsilon) \prod_{p \mid d} \frac{1-1 / p}{1-(p / c) / p}
$$




\begin{tabular}{|rrc|}
\hline$x$ & $H^{*}(x)$ & $8 H^{*}(x) / x$ \\
\hline 1000000 & 97521 & 0.78017 \\
10000000 & 990162 & 0.79213 \\
20000000 & 1988884 & 0.79555 \\
30000000 & 2976321 & 0.79369 \\
40000000 & 3984781 & 0.79696 \\
50000000 & 4987508 & 0.79800 \\
60000000 & 5987504 & 0.79833 \\
70000000 & 6987254 & 0.79854 \\
80000000 & 7972707 & 0.79727 \\
90000000 & 8997355 & 0.79976 \\
100000000 & 10010538 & 0.80084 \\
200000000 & 20090934 & 0.80364 \\
300000000 & 30153902 & 0.80410 \\
400000000 & 40367003 & 0.80734 \\
500000000 & 50551652 & 0.80883 \\
600000000 & 60651064 & 0.80868 \\
700000000 & 70801346 & 0.80916 \\
800000000 & 80950648 & 0.80951 \\
900000000 & 91082121 & 0.80962 \\
1000000000 & 101284007 & 0.81027 \\
\hline
\end{tabular}

TABLE 6. Values of $H^{*}(x)$ for $p \equiv 1 \bmod 4$.

infinitely often. Thus, if $\Delta$ is a prime and $\Delta \equiv 5$ $\bmod 8$, we would have

$$
\mathrm{LLI}<\frac{4}{3}(1-\varepsilon)
$$

infinitely often. Also, if $\Delta$ is a prime and $\Delta \equiv 1$ $\bmod 8$, we would have

$$
\mathrm{ULI}>\frac{1}{2}(1-\varepsilon)
$$

infinitely often. Assuming that the size of $L\left(1, \chi_{\Delta}\right)$ and $h$ are independent, this result (together with the Cohen-Lenstra heuristics) suggests that we'd have

$$
R>(1-\varepsilon) \frac{1}{4} c_{2} \sqrt{\Delta} \log \log \Delta
$$

infinitely often. Figure 1 plots the frequency distribution of the values of

$$
Z=\frac{R}{\sqrt{\Delta} \log \log \Delta}
$$

for all prime values of $\Delta \equiv 1 \bmod 8$, where $8 \times$ $10^{8}<\Delta<10^{9}$. The vertical line on this figure intersects the $Z$ axis at $\frac{1}{2} c_{2}$. Notice that a small but not insignificant portion of the frequency distribution is to the right of this line. The results of [Joshi 1970] are not as good as the extreme values suggested by the truth of the ERH, and Figure 1 provides some evidence that a better result than (5.2) might hold; thus, it is of some interest to conduct a numerical investigation into how large (small) the ULI (LLI) values can be.

Shanks tested (5.1) by attempting to produce values of $\Delta$ for which he might have locally extreme values for the LLI and ULI. For example, if $\Delta \equiv 5 \bmod 8$ and $(\Delta / q)=-1$ for all of the small primes $q$ less than some bound $p$, then we would expect by $(1.3)$ that $L\left(1, \chi_{\Delta}\right)$ would be small. On the other hand, if $\frac{1}{4} \Delta \equiv 7 \bmod 8$ and $(\Delta / q)=1$ for all the primes $q \leq p$, then we would expect $L\left(1, \chi_{\Delta}\right)$ to be large. Shanks made use of Lehmer's numerical sieving device, the DLS-157, to find such special values of $\Delta$. He found no ULI larger than 1 ; in fact, the largest ULI that he found was .7333. Also, he found only a few LLI's less than 1 (these occurred for small values of $\Delta$ only). The values of the LLI's tended to remain stable on average,

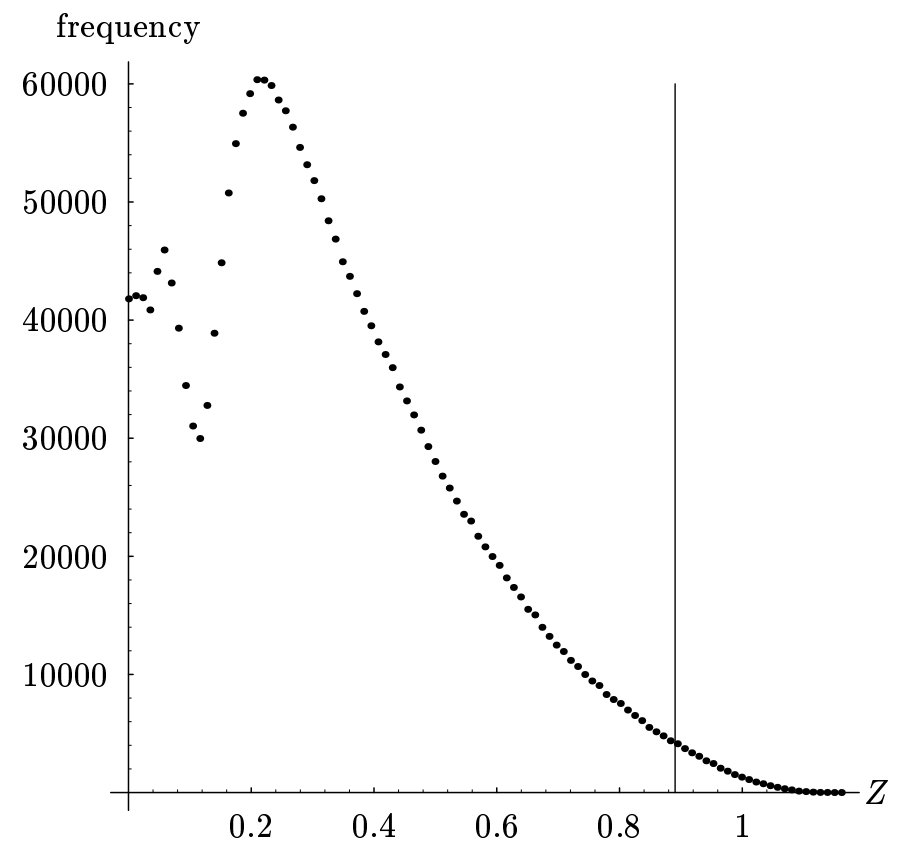

FIGURE 1. Frequency values of $Z$ for $\Delta=p$, with $p \equiv 1 \bmod 8$ prime in the range $8 \times 10^{8}<p<10^{9}$. 
or change very slowly; whereas the ULI's tended to increase very slowly for these special $\Delta$ values; thus, these numerical trials lend support to (5.1).

We used a new sieving device, the MSSU, to extend Shanks' computations. As this instrument has been described in some detail elsewhere [Lukes et al. 1995; Lukes et al. a], we will only mention here that it conducts its search for the kind of numbers that we sought at the rate of over $4 \times 10^{12}$ per second, a considerably faster search rate than that of the DLS-157. For $D \equiv 5 \bmod 8$, we found all values of $D$ such that $0<D<10^{19}$ and $(D / q)=-1$ for $q=3,5,7, \ldots, 199$. For $D \equiv 1 \bmod 8$ we found all the values of $D$ such that $0<D<4 \times 10^{19}$ and $(D / q)=1$ for $q=3,5,7, \ldots, 199$ and for $D \equiv 6 \bmod 8$ and $D \equiv-1 \bmod 4$ we found all the values of $D$ such that $0<D<10^{19}$ and $(D / q)=1$ for $q=3,5,7, \ldots, 199$. We evaluated the class number, regulator, and $L\left(1, \chi_{\Delta}\right)$ for each of the several thousand numbers that resulted by using the Shanks heuristic [Mollin and Williams, p.283]. We then selected the " $L\left(1, \chi_{\Delta}\right)$-lochamps" and "LLI-lochamps" from the values of $D \equiv 5$ mod 8 , namely those $D$ with the property that their corresponding $L\left(1, \chi_{\Delta}\right)$ value (or LLI value) is less than that of any smaller $D$. From each of the other sets of $D$ values we selected the " $L\left(1, \chi_{\Delta}\right)$ hichamps" and "ULI-hichamps," those $D$ with the property that their corresponding $L\left(1, \chi_{\Delta}\right)$ value (or ULI value) is greater than that of any smaller $D$ in the same set. For these $D$ with the most extreme $L\left(1, \chi_{\Delta}\right)$, LLI, and ULI values we computed $h, R$, and $L\left(1, \chi_{\Delta}\right)$ using the techniques of Sections 2 and 3 . In every case the results were the same as those produced by the Shanks heuristic. The largest ULI we found is ULI $=0.741429825 \ldots$ (with $L\left(1, \chi_{\Delta}\right)=4.98741315 \ldots, h=2$ ), for

$$
D=2323617473234474719 \text {. }
$$

The least LLI we found is LLI $=1.24745080 \ldots$ (with $\left.L\left(1, \chi_{\Delta}\right)=0.158960540 \ldots, h=4\right)$, for

$$
D=18974003020179917 .
$$

Since the techniques of Sections 2 and 3 for computing $h$ require the truth of the $\mathrm{ERH}$, the fact that both these techniques and the Shanks heuristic give the same results increases our confidence that the computed values are correct, even if the $\mathrm{ERH}$ is false. Also, the Shanks heuristic is much faster than the method of Sections 2 and 3, so it provided us with a relatively quick way to examine all the numbers produced by the sieve. Even if the class numbers computed by the Shanks heuristic are wrong, they will still be very close to the actual value, and their corresponding $L\left(1, \chi_{\Delta}\right)$ values will be quite accurate. At any rate, we would only expect the Shanks heuristic to give erroneous results for very large class numbers which, by the CohenLenstra heuristics [Cohen and Lenstra 1984], are extremely rare.

Following Shanks we define the symbols $a R_{p}$ and $\left(a N_{p}\right)$ to represent the least integers congruent to $a$ modulo 8 such that

$$
\left(\frac{a R_{p}}{q}\right)=1 \quad \text { and } \quad\left(\frac{a N_{p}}{q}\right)=-1
$$

for all odd primes $q \leq p$. We computed tables of $a R_{p}$ for $a=3,6,7$ and $a N_{p}$ for $a=5$. We also computed similar tables of $a R_{p}$ and $a N_{p}$ when we added the extra constraint that $a R_{p}$ and $a N_{p}$ be prime. We provide example tables here for the combined results for the prime values of $3 R_{p}$ and $7 R_{p}$ and for the prime values of $5 N_{p}$, together with the ULI and LLI values. Corresponding tables for $a=1$ can be found in the supplementary pages to [Lukes et al. a]. Notice that the tendency for the ULI's is to very slowly increase and for the LLI's is to remain stable with minor fluctuations about $\frac{4}{3}$. These tendencies were also displayed in all the other tables. Thus, the results that we have obtained completely support Shanks' earlier findings and therefore support the truth of (5.1). At least, we have not found anything that would lead us to believe that the ERH has been violated.

Although such values of $D$ surely must exist, it seems to be very difficult to produce a value of $D$ with a ULI close to 1 . We attempted to do 


\begin{tabular}{|c|c|c|c|c|c|}
\hline$p$ & $R_{p}$ & $R$ & $h$ & $L(1, \chi)$ & ULI \\
\hline 3 & 7 & 2.76865 & 1 & 1.04645 & 0.488140 \\
\hline 5 & 19 & 5.82893 & 1 & 1.33724 & 0.512241 \\
\hline 7 & 79 & 5.07513 & 3 & 1.71299 & 0.549523 \\
\hline 11 & 331 & 36.25638 & 1 & 1.99283 & 0.567255 \\
\hline 13 & 751 & 57.94214 & 1 & 2.11433 & 0.570617 \\
\hline 17 & 1171 & 25.37280 & 3 & 2.22439 & 0.585134 \\
\hline 19 & 7459 & 73.05341 & 3 & 2.53759 & 0.610832 \\
\hline 23 & 10651 & 270.87206 & 1 & 2.62463 & 0.622710 \\
\hline 29 & 18379 & 367.19773 & 1 & 2.70856 & 0.629349 \\
\hline 31,37 & 78439 & 813.56346 & 1 & 2.90486 & 0.642576 \\
\hline 41 & 399499 & 1890.86355 & 1 & 2.99159 & 0.631650 \\
\hline 43 & 1234531 & 3537.86780 & 1 & 3.18412 & 0.653616 \\
\hline 47,53 & 1427911 & 3841.39768 & 1 & 3.21468 & 0.657630 \\
\hline 59 & 4355311 & 6958.99836 & 1 & 3.33454 & 0.665368 \\
\hline 61 & 5715319 & 8109.80131 & 1 & 3.39226 & 0.673017 \\
\hline 67 & 49196359 & 24407.90384 & 1 & 3.47987 & 0.662406 \\
\hline 71 & 117678031 & 38495.70798 & 1 & 3.54866 & 0.665425 \\
\hline 73 & 180628639 & 49263.42426 & 1 & 3.66548 & 0.682492 \\
\hline 79,83 & 452980999 & 78083.74919 & 1 & 3.66877 & 0.673261 \\
\hline 89,97 & 505313251 & 83941.62341 & 1 & 3.73419 & 0.684123 \\
\hline $101, \ldots, 109$ & 9248561191 & 127289.80150 & 3 & 3.97079 & 0.698473 \\
\hline 113 & 152524816291 & 6690.84067 & 239 & 4.09457 & 0.696458 \\
\hline $113,127,131$ & 348113924239 & 2445102.46006 & 1 & 4.14415 & 0.698553 \\
\hline 137 & 916716646759 & 3976755.53799 & 1 & 4.15347 & 0.693040 \\
\hline 139 & 1086257787619 & 637789.47424 & 7 & 4.28360 & 0.713513 \\
\hline 149 & 4606472154439 & 707977.15943 & 13 & 4.28823 & 0.704162 \\
\hline 151 & 4726529308939 & 9447793.54167 & 1 & 4.34569 & 0.713422 \\
\hline 157 & 35032713351619 & 8533304.31730 & 3 & 4.32515 & 0.697114 \\
\hline $163, \ldots, 179$ & 46257585588439 & 30459726.68748 & 1 & 4.47852 & 0.720076 \\
\hline 181 & 251274765020899 & 23977422.86688 & 3 & 4.53784 & 0.719268 \\
\hline 191 & 316934672172031 & 81024861.17467 & 1 & 4.55127 & 0.720036 \\
\hline., 229 & 2871159201832639 & 246120736.62994 & 1 & 4.59324 & 0.714308 \\
\hline $233, \ldots, 263$ & 632590969227841471 & 3833565622.42494 & 1 & 4.81993 & 0.722316 \\
\hline
\end{tabular}

TABLE 7. $3 R_{p}$ and $7 R_{p}$ : least prime solutions.

this by finding a $D$ value with a large $L\left(1, \chi_{\Delta}\right)$ value. We used an unpublished idea of Lehmer which he employed to find the 20 digit value of $D$ with a small $L(1, \Delta)$ value that appears in [Lehmer et al. 1970, p. 439]. We examined numbers of the form $D=A+B X$, where $B=\prod_{i=j}^{k} p_{i}$, for $p_{i}$ the $i$-th prime, and $\left(A / p_{i}\right)=1$, for $i=j, j+1, \ldots$, $k$. In our case we used $B=271 \cdot 277 \cdot \ldots \cdot 313 \approx$ $5.277 \times 10^{19}$ and the least nonsquare value of $A$. We then employed the MSSU to sieve on values of
$X$ by using as moduli 8 and primes $p_{1}, p_{2}, \ldots, p_{m}$ with $p_{m} \leq 269$ such that $A+X B \equiv 6 \bmod 8$ and

$$
\left((A+X B) / p_{i}\right)=1,
$$

for $i=1,2, \ldots, m$. Henri Cohen used the technique of [Cohen et al. 1993] to evaluate the $L\left(1, \chi_{\Delta}\right)$ values for some of these $D$ values. The largest ULI occurred for

$$
D=13208708795807603033522026252612243246,
$$




\begin{tabular}{|c|c|c|c|c|c|}
\hline$p$ & $N_{p}$ & $R$ & $h$ & $L(1, \chi)$ & LLI \\
\hline 3 & 5 & 0.48121 & 1 & 0.430408 & 0.44355 \\
\hline 5 & 53 & 1.96572 & 1 & 0.540024 & 1.61246 \\
\hline 7,11 & 173 & 2.57081 & 1 & 0.390910 & 1.38799 \\
\hline 13 & 293 & 2.83665 & 1 & 0.331438 & 1.24669 \\
\hline 17 & 2477 & 6.47234 & 1 & 0.260093 & 1.15802 \\
\hline 19,23 & 9173 & 12.47223 & 1 & 0.260446 & 1.24696 \\
\hline 29 & 61613 & 36.23370 & 1 & 0.291948 & 1.51764 \\
\hline $31,37,41$ & 74093 & 7.21597 & 5 & 0.265098 & 1.38758 \\
\hline 43 & 170957 & 16.93918 & 3 & 0.245810 & 1.32491 \\
\hline 47 & 360293 & 68.23691 & 1 & 0.227363 & 1.25504 \\
\hline 53 & 679733 & 92.04349 & 1 & 0.223282 & 1.25592 \\
\hline 59,61 & 2004917 & 48.29722 & 3 & 0.204656 & 1.18549 \\
\hline 67 & 69009533 & 869.69643 & 1 & 0.209383 & 1.31182 \\
\hline 71 & 138473837 & 1369.29769 & 1 & 0.232725 & 1.47713 \\
\hline 73 & 237536213 & 1725.64096 & 1 & 0.223931 & 1.43508 \\
\hline 79 & 384479933 & 2087.35754 & 1 & 0.212907 & 1.37580 \\
\hline 83 & 883597853 & 3018.26471 & 1 & 0.203076 & 1.33041 \\
\hline $89, \ldots, 113$ & 1728061733 & 4021.14004 & 1 & 0.193463 & 1.28086 \\
\hline 127 & 9447241877 & 1252.37753 & 7 & 0.180389 & 1.22431 \\
\hline $131,137,139$ & 49107823133 & 18804.68086 & 1 & 0.169715 & 1.17733 \\
\hline 149 & 1843103135837 & 119080.85359 & 1 & 0.175427 & 1.26915 \\
\hline 151,157 & 4316096218013 & 192239.83257 & 1 & 0.185066 & 1.35078 \\
\hline 163,167 & 15021875771117 & 344898.80858 & 1 & 0.177975 & 1.31520 \\
\hline 173,179 & 82409880589277 & 804942.51462 & 1 & 0.177339 & 1.33146 \\
\hline 181 & 326813126363093 & 1551603.41110 & 1 & 0.171656 & 1.30445 \\
\hline 191,193 & 390894884910197 & 1650908.48845 & 1 & 0.167002 & 1.27101 \\
\hline 197 & 1051212848890277 & 547589.04349 & 5 & 0.168892 & 1.29600 \\
\hline $199,211,223$ & 4075316253649373 & 5291574.72421 & 1 & 0.165780 & 1.28593 \\
\hline 227 & 274457237558283317 & 45653225.95687 & 1 & 0.174286 & 1.39371 \\
\hline 229 & 443001676907312837 & 6097479.67224 & 9 & 0.164899 & 1.32287 \\
\hline 233 & 599423482887195557 & 65388978.22854 & 1 & 0.168914 & 1.35780 \\
\hline 239 & 614530964726833997 & 64783176.97206 & 1 & 0.165280 & 1.32880 \\
\hline $241, \ldots, 263$ & 637754768063384837 & 22908547.79705 & 3 & 0.172116 & 1.38410 \\
\hline
\end{tabular}

TABLE 8. $5 N_{p}$ : least prime solutions.

where $L\left(1, \chi_{\Delta}\right)=5.324999338 \ldots(h=1)$. This is a large $L\left(1, \chi_{\Delta}\right)$, but when we evaluate the ULI we only get ULI $=.669706597 \ldots$

\section{CONCLUSION}

Elliot [Elliot 1969] has shown that if $\varepsilon>0$ is given, then there exist constants $c_{3}$ and $c_{4}$ (depending on $\varepsilon$ ) and a set $S=S(x)$ for $x \geq 2$, such that for all prime values of $\Delta \leq x, \Delta \notin S$, we have

$$
\frac{c_{3}}{\log \log \Delta} \leq L(1, \chi) \leq c_{4} \log \log \Delta .
$$

Furthermore, $S$ has cardinality at most $O\left(x^{\varepsilon}\right)$. In view of the Cohen-Lenstra heuristics and the numerical evidence presented above, this would seem to permit us to conjecture that there exists an infinite set of values of $\Delta$ for which

$$
R \gg \frac{\sqrt{\Delta}}{\log \log \Delta}
$$


In fact it even appears that there must exist an infinite set of values of $\Delta$ such that

$$
R \gg \sqrt{\Delta} \log \log \Delta .
$$

At present the best result of this type is that of Halter-Koch [Halter-Koch 1989] where it is shown that there exists an infinite set of values of $\Delta$ such that

$$
R \gg \log ^{4} \Delta \text {. }
$$

This result is so much worse than (6.1) that it should be possible (without appealing to the ERH or the Gauss Conjecture) to get a better result than (6.2).

\section{REFERENCES}

[Bach 1994] E. Bach, "Improved approximations for Euler products", unpublished manuscript, 1994.

[Chowla 1949] S. Chowla, "Improvement of a theorem of Linnik and Walfisz", Proc. London Math. Soc. 50 (1949), 423-429.

[Cohen et al. 1993] H. Cohen, F. Diaz y Diaz, and M. Olivier, "Calculs de nombres de classes et de régulateurs de corps quadratiques en temps sousexponentiel", pp. 35-46 in Séminaire de Théorie des Nombres de Paris 1990-1991, Progress in Math. 108, Birkhäuser, Boston, 1993.

[Cohen and Lenstra 1983] H. Cohen and H. W. Lenstra, Jr., "Heuristics on class groups", pp. 2636 in Number Theory, CUNY, 1982 (edited by D. V. Chudnovsky), Lecture Notes in Math. 1052, Springer, New York, 1983.

[Cohen and Lenstra 1984] H. Cohen and H. W. Lenstra, Jr., "Heuristics on class groups of number fields", pp. 33-62 in Number Theory, Noordwijkerhout, 1983 (edited by H. Jager), Lecture Notes in Math. 1068, Springer, New York, 1984.

[Elliot 1969] P. D. T. A. Elliot, "On the size of $L(1, \chi)$ ", J. reine angew. Math. 236 (1969), 26-36.

[Halter-Koch 1989] F. Halter-Koch, "Reell-quadratischer Zahlkörper mit größer Grundeinheit", Abh. Math. Sem. Univ. Hamburg 59 (1989), 171-181.
[Hooley 1984] C. Hooley, "On the Pellian equation and the class number of indefinite binary quadratic forms", J. reine angew. Math. 353 (1984), 98-131.

[Hua 1982] L. K. Hua, Introduction to Number Theory, Springer, New York, 1982.

[Joshi 1970] P. T. Joshi, "The size of $L(1, \chi)$ for real nonprincipal residue characters $\chi$ with prime modulus", J. Number Theory 2 (1970), 58-73.

[Landau 1936] E. Landau, "On a Titchmarsh-Estermann sum", J. London Math. Soc. 11 (1936), 242245.

[Lehmer et al. 1970] D. H. Lehmer, E. Lehmer, and D. Shanks, "Integer sequences having prescribed quadratic character", Math. Comp. 24 (1970), 433451.

[Lenstra 1982] H. W. Lenstra, Jr., "On the Calculation of Regulators and Class Numbers of Quadratic Fields", pp. 123-150 in Number Theory Days, Exeter, 1980, London Math. Soc. Lecture Note Series 56, Cambridge U. Press, Cambridge, 1982.

[Littlewood 1928] J. E. Littlewood, "On the class number of the corpus $P(\sqrt{ }-k)$ ", Proc. London Math. Soc. 27 (1928), 358-372.

[Lukes et al. 1995] R. F. Lukes, C. D. Patterson, and H. C. Williams, "Numerical Sieving Devices: Their History and Some Applications", Nieuw Archief voor Wiskunde (4) 13 (1995), 113-139.

[Lukes et al. a] R. F. Lukes, C. D. Patterson, and H. C. Williams, "Some results on pseudosquares", to appear in Math. Comp.

[Mollin and Williams] R. A. Mollin and H. C. Williams, "Computation of the class number of a real quadratic field", Utilitas Math. 41 (1992), 259-308.

[Nagell 1922] T. Nagell, "Zur Arithmetik der Polynome", Abh. Math. Sem. Univ. Hamburg 1 (1922), 179-194.

[Oesterlé 1979] J. Oesterlé, "Versions effectives du théorème de Chebotarev sous l'hypothése de Riemann généralisée", pp. 165-167 in Journées arithmétiques, Luminy, 1978, Astérisque 61, Soc. math. de France, Paris, 1979.

[Shanks 1971] D. Shanks, "Class number, a theory of factorization and genera", pp. $415-440$ in Number 
Theory Institute, Stony Brook, 1969, Proc. Symp. Pure Math. 20, Amer. Math. Soc., Providence, 1971.

[Shanks 1973] D. Shanks, "Systematic examination of Littlewood's bounds on $L(1, \chi)$ ", pp. 267-283 in Analytic number theory, St. Louis, 1972 (edited by
H. G. Diamond), Proc. Symp. Pure Math. 24, Amer. Math. Soc., Providence, 1973.

[Stephens and Williams 1988] A. J. Stephens and H. C. Williams, "Computation of real quadratic fields with class number one", Math. Comp. 51 (1988), 809-824.

Michael J. Jacobson, Jr., Department of Computer Science, University of Manitoba, Winnipeg, Manitoba, Canada R3T 2N2 (jacobs@cs.uni-sb.de)

Richard F. Lukes, Department of Computer Science, University of Manitoba, Winnipeg, Manitoba, Canada R3T 2N2 (rflukes@cs.umanitoba.ca)

Hugh C. Williams, Department of Computer Science, University of Manitoba, Winnipeg, Manitoba, Canada R3T 2N2 (Hugh_Williams@csmail.cs.umanitoba.ca)

Received January 4, 1995; accepted in revised form August 8, 1995 